\title{
Doing More with Less: Price Discrimination with Three-part vs Two-part tariffs
}

\author{
Adib Bagh Hemant K. Bhargava \\ Department of Economics Graduate School of Management \\ $\{$ abagh, hemantb\}@ucdavis.edu \\ University of California Davis
}

November 21, 2007

\begin{abstract}
We show that a relatively small menu of three-part tariffs (3PTs) can be more profitable and, sometimes, socially more desirable than a larger menu (more items) of two-part tariffs (2PTs). Often, a single three-part tariff can beat a sorting menu comprising multiple two-part tariffs. Moreover, this 3PT menu can be designed with less information about consumer preferences, relative to the menu of two-part tariffs which, in order to segment customers optimally, needs fine-grained information about preferences. Our analysis reveals a counterintuitive insight that more ornamental tariffs need not always be more profitable, the source of ornateness influences how efficiently the tariff can price discriminate. The 3PT structure not only produces higher profit, but has lower managerial and decision complexity.
\end{abstract}

Keywords. Asymmetric information, multi-part tariffs, Information technology 


\section{Management Insight Paragraph}

Multi-part tariffs, such as menus of two-part tariffs and three-part tariffs, are widely used in industry, especially for pricing of information goods, online services, telecommunications products, etc. This paper examines the effectiveness of these price structures for price discriminating between heterogeneous customers. We show that a relatively small menu of three-part tariffs (3PTs) can be more profitable and, sometimes, socially more desirable than a larger menu (more items) of two-part tariffs (2PTs). Often, a single three-part tariff can beat a menu of multiple two-part tariffs. Moreover, this 3PT menu has lower "hidden costs" - it can be designed with less information about consumer preferences, relative to the menu of two-part tariffs. The 3PT structure not only produces higher profit, but has lower managerial and decision complexity. 

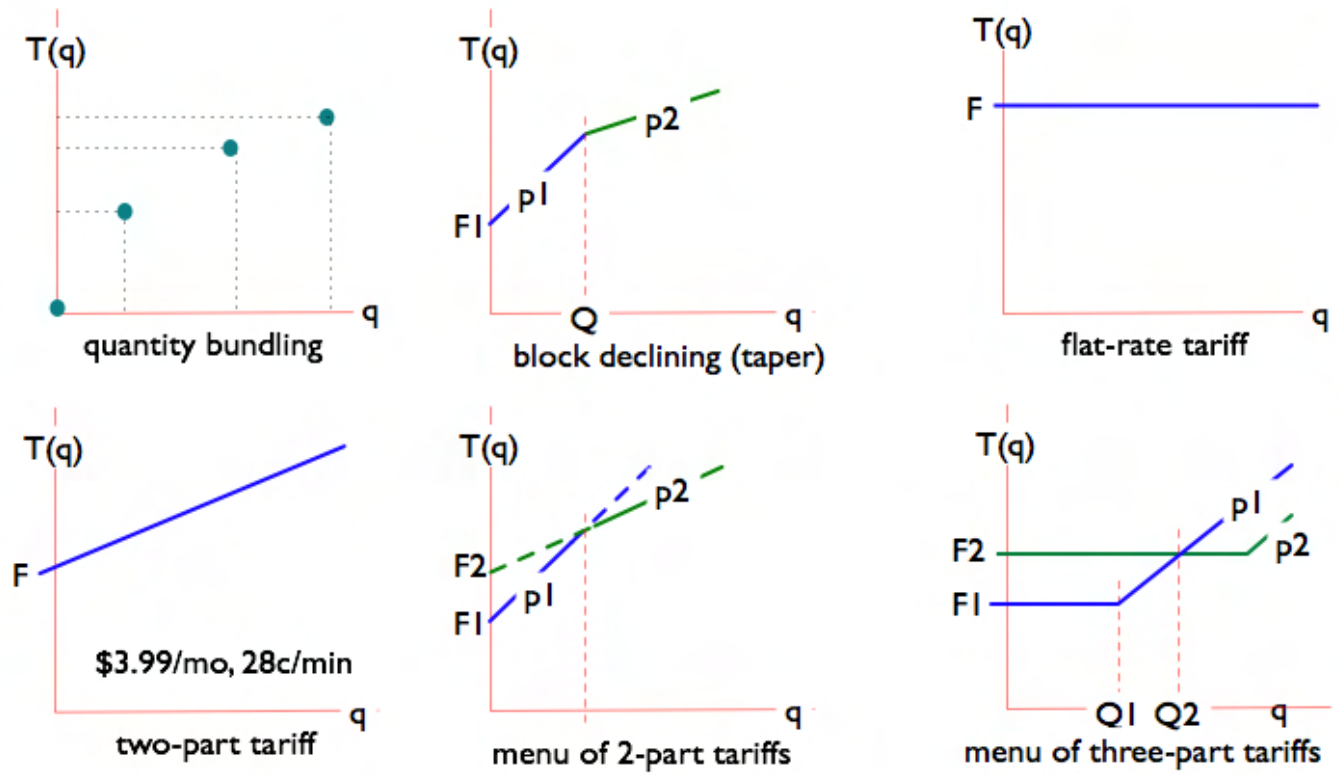

Figure 1: Payment schedule under various nonlinear pricing schemes.

\section{Introduction}

Markets for information technology and telecommunications products and services feature a wide array of nonlinear price structures. This helps the firm in price discriminating between heterogeneous consumers by inducing them to self-select due to differences both in expected consumption amount and willingnessto-pay for each unit. Quantity-price bundling is the most general implementation of nonlinear pricing, but frequently firms use mechanisms such as block pricing (which specifies intervals over which the unit price remains constant) and multi-part tariffs such as flat-rate pricing, two-part tariffs, three-part tariffs (Leland and Meyer, 1976). In the latter case, the firm often offers a menu of multi-part tariffs in order to improve its ability to price discriminate. Figure 1 graphically depicts the payment schedule, as a function of consumption quantity, under various schemes for nonlinear pricing. Table 1 gives real-world examples of flat-rate pricing, two-part tariffs, three-part tariffs, block-declining tariffs, and quantity-price bundling.

This paper analyzes menus of multi-part tariffs (MPT, for short), specifically menus of two-part tariffs (2PT) and three-part tariffs (3PT) respectively. While MPTs generate, in principle, lower revenue than the theoretically optimal nonlinear price schedule (quantity-price bundling), the revenue gap is often 
Table 1: Examples of non-linear pricing structures.

\begin{tabular}{|c|c|c|}
\hline Tariff Structure & Product & Example \\
\hline $\begin{array}{l}\text { Flat-rate } \\
\text { (unlimited use) }\end{array}$ & $\begin{array}{l}\text { Internet access } \\
\text { Music download }\end{array}$ & $\begin{array}{l}\text { SBC: } \$ 17.99 / \mathrm{mo} \\
\text { Rhapsody: } \$ 9.99 / \mathrm{mo}\end{array}$ \\
\hline $\begin{array}{l}\text { Menu of two-part } \\
\text { tariffs }\end{array}$ & $\begin{array}{l}\text { International phone calls } \\
\text { (U.S. to India) }\end{array}$ & $\begin{array}{l}\text { AT\&T: } \$ 2.95 / \mathrm{mo} \text { plus } \$ 0.39 / \text { minute; or } \\
\$ 3.99 / \text { mo plus } \$ 0.28 / \text { minute. }\end{array}$ \\
\hline $\begin{array}{l}\text { Menu of three-part } \\
\text { tariffs }\end{array}$ & Cell phone service & $\begin{array}{l}\text { Verizon: }(\$ 40 / \mathrm{mo}, 450 \text { minutes, } \$ 0.45 / \mathrm{min}) \text { or } \\
(\$ 80 / \mathrm{mo}, 1350 \text { minutes, } \$ 0.35 / \mathrm{min}) \text {. }\end{array}$ \\
\hline $\begin{array}{l}\text { Menu of three-part } \\
\text { tariffs }\end{array}$ & Data center hosting & $\begin{array}{l}\text { RimuHosting: } \quad(\$ 20 / \mathrm{mo}, 30 \mathrm{~GB}, \quad \$ 1 / \mathrm{GB}) \\
\ldots(\$ 50 / \mathrm{mo}, 75 \mathrm{~GB}, \$ 1 / \mathrm{GB}) .\end{array}$ \\
\hline $\begin{array}{l}\text { Block-declining tar- } \\
\text { iff (taper) }\end{array}$ & Parking garage & $\$ 6 / \mathrm{hr}$ for first 2 hours; $\$ 4 / \mathrm{hr}$ thereafter. \\
\hline Quantity discount & Firewire drive & $\begin{array}{l}\text { SmartDisk: } \$ 130 \text { for } 40 \mathrm{~GB} ; \quad \$ 160 \text { for } 60 \mathrm{~GB} \text {; } \\
\$ 180 \text { for } 80 \mathrm{~GB} \text {. }\end{array}$ \\
\hline
\end{tabular}

small in practice (Wilson, 1993; Miravete, 1996). Often, the incremental overhead cost of quantityprice bundling exceeds its revenue advantage over MPTs. Moreover, the fixed fee component of MPTs is attractive to sellers when they incur per-customer transaction costs even when customers have little or no usage (Sundararajan, 2004). These tariff structures are also more appropriate for products with longterm usage contracts because, unlike a predefined menu of price-quantity bundles, the price structure typically has a variable fee for "above-anticipated" (or "above-allowance") consumption. Due to these characteristics - lower complexity (easier for consumers to understand, simpler for firms to administer) and flexibility in consumption quantity - MPTs are widely used in practice. However, despite a large literature on their application, crucial aspects of these tariff structures are not well-understood, including their comparative performance and tariff optimization for price discrimination.

The salient challenge for a firm that tries to price discriminate amongst a population of heterogeneous consumers is how finely to tune its discrimination strategy. In a recent survey of research on price discrimination, Armstrong (2006) notes that a tariff structure $A$ is more ornate or more fine-tuned than structure $B$ if either $A$ requires more information about the consumers (for example more detailed information about the nature and distribution of preferences), or $A$ employs more instruments than $B$. For example, a 2PT uses more instruments, and hence it is more ornate, than a linear price or a flat-rate tariff. Similarly a $3 \mathrm{PT}$ is more ornate than a $2 \mathrm{PT}$. And a menu of, say, five $2 \mathrm{PT}$ (ten instruments) is more ornate than a single $3 \mathrm{PT}$ (three instruments). Armstrong then makes the following intuitive 
observation: a more ornate tariff must be at least as profitable as one less ornate (before considering decision or administrative costs of fine-tuning), and that the efficiency loss under monopoly pricing is typically ascribed to the firm's inability to deploy a sufficiently ornate tariff.

In this paper we present, in the context of multi-part tariffs, a counterpoint to this intuitive understanding that a more ornate or more discriminating tariff structure produces higher profits for the firm. In fact, we show that a single 3PT designed using limited information about the consumers can, depending on the degree of consumer heterogeneity in the market, do better (in terms of both profits and social welfare) than a large menu of $2 \mathrm{PTs}$ that employs more instruments and more information about the consumers. This result indicates that the source of a tariff's ornateness affects how well it can price discriminate: a 2PT menu has more menu items but it loses degrees of freedom due to consumer self-selection constraints, while a three-part tariff offers the firm an additional independent dimension for separating heterogeneous consumers. Thus, three-part tariffs are more efficient and effective at price discrimination. This leads to better extraction of consumer surplus despite using less information and fewer instruments compared to longer menus (with more items) of 2PTs. We will also show that although this type of efficiency leads to higher profits, it may or may not lead to higher total surplusthe surprising element of this result is that higher consumer and social surplus is a possible adjunct of increased extraction of consumer surplus. In addition to these comparative properties of the tariff structures, we also specify the optimal form of a menu of two-part and three-part tariffs, respectively.

Out of the vast and growing literature on MPTs and block pricing (Clay et al., 1992; Miravete, 1996; Wilson, 1993; Masuda and Whang, 2006), the one most closely related to our work is Kolay and Shaffer (2003) who compare price-quantity bundling to a 2PT menu (which, incidentally, is equivalent to $N$-part block-declining tariffs (Faulhaber and Panzar, 1977)). Not surprisingly, they find that in the case of a market with two types of consumers, a price-quantity bundling menu of two items is more profitable than a two-part menu of two items. Moreover, they find that the relative efficiency of these two menus (i.e. whether or not one menu yields more social surplus than other) is dependent on the functional forms of the demand function. This result is independent of the distribution of the types as long as we assume that both types are served. Our analysis compares a menu of two-part tariffs with three-part tariffs, a tariff structure that is equally common in practice and easy to implement. 
Clearly, a menu of $n$ three-part tariffs will be no less profitable than a menu of $n$ two-part tariffs. The surprising aspect of our result is that a three-part menu of $i$ items can yield more profits than a menu of $n$ two-part tariffs even when $i$ is strictly less than $n$. The significance of this result is that the three-part tariff not only has higher net profits, but in addition has lower "hidden costs" of tariff optimization and segment management: the more ornate 2PT menu requires more detailed information about consumer preferences, and implementing it has higher costs of advertising and managing multiple segments each targeted with a unique menu item.

\section{Modeling Framework and Illustration of Results}

In this section we present a series of examples to illustrate our key results, the intuition behind the results, and the data assumptions underlying these results. We model consumer heterogeneity via a single type parameter as a proxy for differences in marginal valuation. Let $I$ denote the number of distinct customer segments, and $\lambda_{i}(i=1 \ldots I)$ the size of segment $i$. Each customer in segment $i$ has a demand function $D_{i}(p)$, and for each $p$ a type $i+1$ customer has higher demand than a type $i$ customer. Let $\pi_{2}^{*}$ denote the profit under the optimal menu with two-part prices, and $\pi_{3}^{*}$ the profit with a single three-part tariff. Note that in general the optimal 2PT menu may have some pooling. Our examples cover the complete-sorting case (which should be the hardest for a single three-part tariff to beat) and we also demonstrate the insights under partial sorting solutions. When the 2PT menu causes some pooling, it is useful to distinguish between the exogenous distribution (the $\lambda$ 's) and the endogenous distribution of segments or pools of customers that pick the same item off the menu.

While our results are robust to the form of the individual demand function, all examples use a linear demand function $D_{i}(p)=\frac{a_{i}-p}{b_{i}}$ for ease of computation. Example 1 illustrates the main result-a single three-part tariff beats a larger, optimal, menu of two-part tariffs—under a simplistic setting with just two customer types and linear demand. Later examples demonstrate the extension to multiple customer types, and illustrate the requirement (left-skewed distribution, i.e., sufficiently more low type customers) that underlies the result. Example 2 demonstrates our second result, that the superior single three-part tariff can be designed using less information than needed for the optimal 2PT menu. Example 3 then further generalizes the condition underlying the result: left-skewness is not required in the exogenous 
distribution of preferences, it need only be a characteristic of the optimal 2PT menu (i.e., sufficiently large fraction of consumers should pick the "cheaper" item from the 2PT menu). Finally, Example 4 presents a generalization of the main result-under certain conditions the superior 3PT structure may need to have more than one item-and relates this structure to the design of the optimal 2PT menu.

Starting with the simple case $I=2$ (later examples allow arbitrarily large $I$ ), we write $\lambda_{1}=\lambda$, with $\lambda_{2}=(1-\lambda)$. The ratio of type 1 (to type 2 ) customers is $\frac{\lambda}{1-\lambda}$. Let $\hat{\lambda}$ be the (minimum) threshold of type 1 customers for complete sorting to be optimal. For $\lambda<\hat{\lambda}$, type 1 customers are priced out under the optimal menu of two-part tariffs, consequently this design is dominated by the single three-part tariff. Therefore the interesting region for analysis is $\lambda>\hat{\lambda}$.

Example 1 Let $I=2$, with $D_{1}(p)=(100-p)$, and $D_{2}(p)=\frac{5}{4}(200-p)$. We have $\hat{\lambda}=0.77$, and $\pi_{3}^{*}>\pi_{2}^{*}$ for all $\lambda>\hat{\lambda}$. For instance, consider $\lambda=0.8$. The optimal solution under two-part pricing is

$$
\left(F_{1}, p_{1}\right)=(2093.43,35.3) ; \quad\left(F_{2}, p_{2}\right)=(10138.4,0) ; \quad \pi_{2}^{*}=5529.41
$$

while the optimal three-part tariff is

$$
(F, Q, p)=(4914.93,86.96,65.22) ; \quad \pi_{3}^{*}=5978.26
$$

The single three-part tariff produces $8 \%$ higher profit than the optimal menu of two-part tariffs. ${ }^{1}$

Example 1 demonstrates that the single 3PT is (from the firm's point of view) more efficient at extracting consumer surplus despite being more austere in structure. It yields higher profit than the menu of two-part tariffs for all $\lambda$ for which the $2 \mathrm{PT}$ serves both types. ${ }^{2}$ The result need not always hold. For example, changing $a_{2}$ from 200 to 150 shows that the single 3PT outperforms the 2PT menu only when the density of low-type consumers is sufficiently large $(\lambda>0.656$, which can alternately be written as $\frac{\lambda}{1-\lambda}>\approx 2$ ); the $2 \mathrm{PT}$ menu dominates for $\lambda \in[0.57,0.656]$. This illustrates a more general point formalized later in Theorems 1 and 3: the single three-part tariff outperforms the menu of

\footnotetext{
${ }^{1}$ The profit advantage of the single 3PT is even higher under different parameter settings. The parameters used in the example were chosen to demonstrate several effects beyond the profit difference.

${ }^{2}$ Recall that if $\lambda$ is too small then the two-part menu would ignore the low type customers, trivially ensuring that the $3 \mathrm{PT}$ does as well as the non-sorting $2 \mathrm{PT}$ menu.
} 
two-part tariffs so long as the distribution of consumer types is sufficiently skewed towards the low-type consumers. The sufficiency requirement is quite realistic because many markets have a greater mass of consumers at the lower end. The concept easily generalizes when there is a larger number of consumer segments, as shown in the remaining examples.

Example 2 Assume $I=3, D_{i}=a_{i}-p$, with $a_{i}=i$, and weights $\lambda_{1}=.8, \lambda_{2}=0.18$, and $\lambda_{3}=0.02$. With these parameters, the optimal 2PT menu has 3 items, one targeted to each type: $\left(F_{1}^{*}=0.47, p_{1}^{*}=0.25\right) ;\left(F_{2}^{*}=0.534, p_{2}^{*}=0.11\right) ;$ and $\left(F_{3}^{*}=0.86, p_{3}^{*}=0\right)$. The optimal 3PT menu with single item $(F=0.493, Q=0.88, p=0.56)$ with profit $\pi_{3}^{*}=0.57$, which beats the $2 P T$ menu profit $\pi_{2}^{*}=0.526$.

In Example 2, a single 3PT beats the fully-sorting optimal menu of three 2PTs. The result also holds when the optimal 2PT design involves some pooling. To see this, consider an increase in $a_{3}$. Then for $3 \leq a_{3}<4.25$, the optimal $2 \mathrm{PT}$ will continue to be completely separating. For this entire range, the same single 3PT ( $F=0.493, Q=0.88, p=0.56)$ outperforms any fully separating $2 \mathrm{PT}$ menu with three items. For $4.25<a_{3}<6.8$, the optimal 2PT will consist of only two items, because it pools consumers 1 and 2. For this range too, the single 3PT $(F=0.493, Q=0.88, p=0.56)$ dominates the optimal 2PT menu. ${ }^{3}$ Notice that while the precise shape of the 2PT menu varies according to the problem parameters, the same 3PT design outperforms the optimal 2PT menu in all of these situations.

This analysis demonstrates a notable property: the single 3PT uses fewer instruments and it uses less information than the optimal 2PT menu (it is less ornate in both aspects of Armstrong's definition). More specifically, the superior 3PT $(F=0.493, Q=0.88, p=0.56)$ can be designed knowing only that $a_{3}$ is within some range (here, $3<a_{3}<6.8$ ) whereas the optimal 2PT menu design requires knowing the exact value of $a_{3}$. In fact we show (in $\S 4$ ) that when the firm faces an even greater number of segments, it can design the (shorter) superior 3PT tariff even when it has only partial information about the "tail" of the distribution of types, whereas designing the optimal 2PT requires detailed information about all consumer types. This property is particularly significant from a managerial perspective due to "hidden costs" of price discrimination. With a menu of $2 \mathrm{PTs}$, the firm screens customers by targeting them

\footnotetext{
${ }^{3}$ For $a_{3}>6.8$, the optimal menu will consist of serving the third consumer only. In this case, the optimal 2PT and the optimal 3PT are the same, and they both ignore the lowest two consumers and they both extract full surplus from consumer 3.
} 


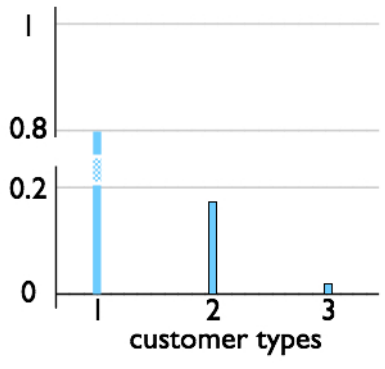

(a) Example 2 .

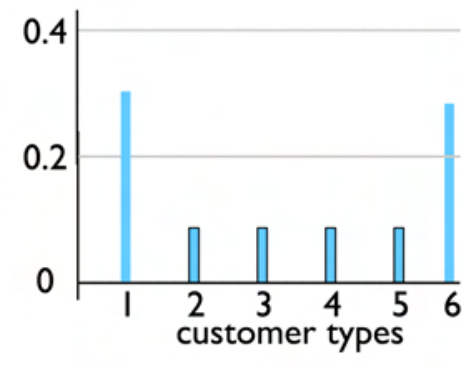

(b) Example 3 (raw data).

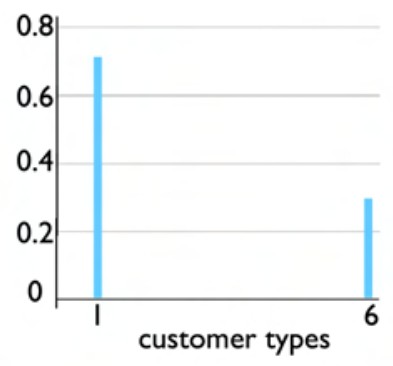

(c) Example 3 (after pooling).

Figure 2: Distribution of customer types. Leftward skewness is exhibited by the exogenous preference data in Example 2, but not in Example 3. However, the optimal 2PT menu in Example 2 pools types $1-5$, and the resulting distribution (of customers that pick the same item from the menu) is skewed to the left.

with distinct offers. But to develop the optimal menu the firm must collect fine-grained information about consumer types (which involves the expense of extensive market research or purchase of customer data), and implementing the menu incurs the cost of advertising and managing multiple segments (see Dhebar (1993) for a nice illustration of these issues in a management case on product versioning). The 3PT design, on the other hand, requires only an approximate understanding of customer preferences and distributions, which managers often intuitively possess, so that the superior 3PT can be implemented with minimal hidden costs.

The previous examples exhibited sufficient leftward-skewness in the exogenous distribution of types (e.g., $\lambda_{1}=0.8$ in Example 1-2). Figure 2(a) graphically depicts the distribution for Example 2. Sometimes the exogenous distribution may not be so skewed (see Example 3 below, depicted in Figure 2(b)), yet the segments created endogenously by a $2 \mathrm{PT}$ menu may exhibit the left-skewed property (i.e., far more buyers pick the "less expensive" tariff, see Figure 2(c)). Our result holds even in such situations. Here, the optimal 2PT menu involves only partial sorting, with fewer items than the number of ex ante segments. Proving the superiority of a single 3PT requires simply showing that it dominates an adjusted demand function (the actual profit under this 3PT is higher than the profit for the adjusted function).

Example 3 Suppose there are six types of customers with demand functions $D_{i}(p)=a_{i}-p$, with $a_{i+j}=a_{i}+j \gamma . \lambda_{1}=0.31, \lambda_{6}=0.29$ while the remaining segments are each $10 \%$ of the market. Then the optimal menu of two part tariffs involves pooling: it has one item targeted to types 1 through 5, and 


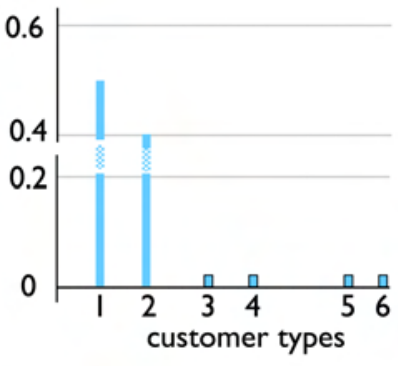

(a) Example 4(1).

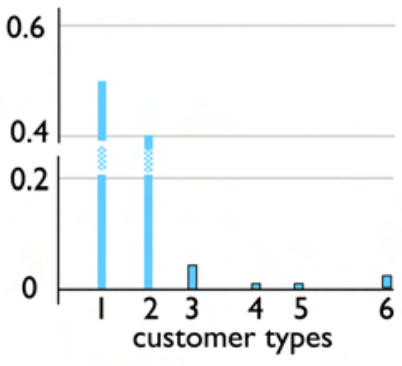

(b) Example 4(2).

Figure 3: Distribution of customer types. Type 1 alone does not have sufficient weight; but combining the weights on types 1 and 2 makes the distribution sufficiently left-skewed. The superior 3PT is designed regardless of how types 4-6 are distributed. The same design outpeforms the 2PT menu under both distributions shown in this figure.

a second for type 6. This means that finding the optimal two-part menu is equivalent to assuming that $70 \%$ of the market has demand $D(p)=a_{i}-p$ and the rest has demand $D(p)=a_{6}-p=\left(a_{i}+5 \gamma-p\right)$. As shown in Examples 1-2, we can beat this two-item menu with a single three-part tariff.

The "leftward skewed" requirement we have employed may be measured by partitioning the distribution (into two parts) at customer type $i$ (for some $i<I$ ), and computing

$$
H(i)=\frac{\lambda_{i}}{\sum_{j=i+1}^{I} \lambda_{j}}
$$

which represents the mass at $j$ relative to the right tail. We will show that there will always exist an $i=k<I$ and a feasible threshold value $\hat{\alpha}_{k}$ such that if $H(k)>\hat{\alpha}_{k}$ then a 3PT menu with $k$ items will outperform the optimal 2PT menu regardless of its size. Specifically, if $H(1)>\alpha_{1}$ then a single 3PT will beat the optimal 2PT menu (as was the case in all of the examples described thus far). And if the problem parameters do not satisfy $H(1)>\alpha_{1}$, then we simply apply the same test for $j=2$ and higher, in order to find that the smallest 3PT menu size needed to outperform the optimal 2PT menu. For example, if the condition holds for $k=2$ (but not $k=1$ ) then there exists a two-item menu of three part tariffs $\left[\left(F_{1}, Q_{1}, \hat{p}\right) ;\left(F_{2}, Q_{2}, \hat{p}\right)\right]$ which beats the optimal 2 pt menu with $I$ items. The next example illustrates this property.

Example 4 Suppose we have $I>2$ types with with demand functions $D_{i}(p)=a_{i}-p$, with $a_{1}<a_{2}<$ 
$\cdots<a_{I}$. Let $\lambda_{1}=0.50$ and $\lambda_{2}=0.4$. Then a menu of two three-part tariffs of the from $\left(F_{1}, Q_{1}, \hat{P}\right)$, $\left(F_{2}, Q_{2}, \hat{p}\right)$ will beat the optimal menu of two part tariffs. This is true no matter how agents 3 through $I$ are distributed (their weights and $a_{i}$ 's, see Figure 3).

Notice that in Example 4, $H(1)$ is relatively small $\left(H(1)=\frac{0.5}{0.5}=1\right)$ while $H(2)=\frac{0.4}{0.1}=4$ is large. Thus, the distribution is not sufficiently left-skewed when the right tail includes the high-mass type 2 segment, but when the measure is computed at $j=2$ the property is satisfied because the collective mass of types 3-6 is small relative to type 2 . The relative mass and marginal valuations of the high-end customers will determine the size of the optimal 2PT menu (which might be as high as 6), but in all cases the two-item 3PT menu will do better.

\section{Comparing Menus of Two- and Three-part Tariffs}

This section analyzes the case where the seller faces a market with just two distinct consumer segments, $i=1,2$. In this setting, we can demonstrate the potential dominance of a single three-part tariff, over the optimal menu of two-part tariffs, with only minimal restrictions on the demand function (see below).

Let $V_{i}(q)$ be the valuation of a segment $i$ consumer for $q$ units, with $v_{i}(q)=\frac{\partial V_{i}}{\partial q}$ the marginal valuation for the $q^{\text {th }}$ unit. Segment 2 is the one with higher valuations. Let $D_{i}(p)$ be the demand curve for an individual type $i$ customer: this is the (optimal) demand of a type $i$ consumer at unit price $p, D_{i}(p)=q_{i}: v_{i}\left(q_{i}\right)=p$. Let $\bar{p}_{i}$ be the highest unit price that commands positive consumption in segment $i$ : segment $i$ has zero demand for $p>\bar{p}_{i}$ and positive demand for $\bar{p} \leq p_{i}$. At the other extreme, let $e_{i}=D_{i}(c)$ represent type $i$ 's consumption at $p=c$ ("efficient" consumption level). The two consumer segments occur in ratios $\lambda$ and $1-\lambda$ respectively. We need only the standard restrictions that guarantee concavity of the 2PT profit function (see e.g., Maskin and Riley (1984)). We state the restriction in terms of the $v_{i}{ }^{\prime}$ s: the equilvalent statement is that $D_{i}(p)$ is strictly downward sloping with increasing elasticity of demand.

Assumption 1 For each type $i$, the marginal valuation function $v_{i}(q)$ is $(1)$ continuous, differentiable, and decreasing in $q$, with (2) $v_{i+1}(q)>v_{i}(q)$ for all $q$ (type $i$ is a "higher" type or a more "heavy user" than $j$ ). 
For example, the commonly used value function $V(q)=\left(a \cdot q-\frac{b}{2} q^{2}\right)$ (see, e.g., Lambrecht and Skiera (2006)) corresponds to marginal valuations $v(q)=(a-b \cdot q)$, yielding the linear demand curve $D(p)=\frac{1}{b}(a-p)$, with $\bar{p}=a$ and $e=\frac{a}{b}$. To make the analysis non-trivial, we also impose the regularity condition that $\lambda$ is large enough that the optimal 2PT menu serves both types (the threshold $\hat{\lambda}$ is specified below; when it is not met the result is trivial because a single 3PT generalizes a single 2PT).

\subsection{Optimal Menu of Two-Part Tariffs}

Let $(F, p)$ represent a two-part tariff, with fixed fee $F$ and unit fee $p$. The firm's revenue from a customer who consumes $q$ units under this tariff is $T(q)=F+p q$. A type $i$ customer's optimal consumption under this tariff is $q_{i}: v_{i}(q)=p$, giving her a gross surplus (before considering fixed fee)

$$
S_{i}(p)=\int_{p}^{\bar{p}_{i}} D(\tau) d \tau
$$

With a single two-part tariff, the firm can extract all of a type 1 customer's surplus by setting $F=S_{1}(p)$ (and then optimizing on $p$ to get the optimal tariff), however type 2 consumers enjoy a net surplus (see Figure $4(\mathrm{a}))$. The firm can therefore increase profits through price discrimination by offering a menu $\left[\left(F_{1}, p_{1}\right) ;\left(F_{2}, p_{2}\right)\right]$. The optimal design reduces to a single-variable optimization problem (see, e.g., Kolay and Shaffer (2003)). Type 1's surplus is extracted by setting $F_{1}^{*}=S_{1}\left(p_{1}\right)$, while the optimal fixed fee $F_{2}$ binds the incentive compatibility constraint for type 2, so that

$$
F_{2}^{*}=F_{1}+\left(S_{2}\left(p_{2}\right)-S_{2}\left(p_{1}\right)\right)
$$

The outcomes for a menu of two-part tariffs are depicted in Figure 4(b). Trivially, $p_{2}^{*}=c$ (which maximizes $S_{2}$ hence $F_{2}$ ). Now the remaining decision variables $F_{1}$ and $F_{2}$ are functions only of $p_{1}$. Thus, the optimal menu can be designed by choosing $p_{1}^{*}$ as below.

$$
\begin{aligned}
\pi_{2}^{*}\left(p_{1}\right) & =\lambda\left(\int_{p_{1}}^{\bar{p}_{1}} D_{1}(\tau) d \tau+\left(p_{1}-c\right) D_{1}\left(p_{1}\right)\right)+(1-\lambda)\left(\int_{p_{1}}^{\bar{p}_{1}} D_{1}(\tau) d \tau+\int_{c}^{p_{1}} D_{2}(\tau) d \tau\right) \\
p_{1}^{*} & =\arg \max _{p_{1}} \pi_{2}^{*}\left(p_{1}\right)
\end{aligned}
$$




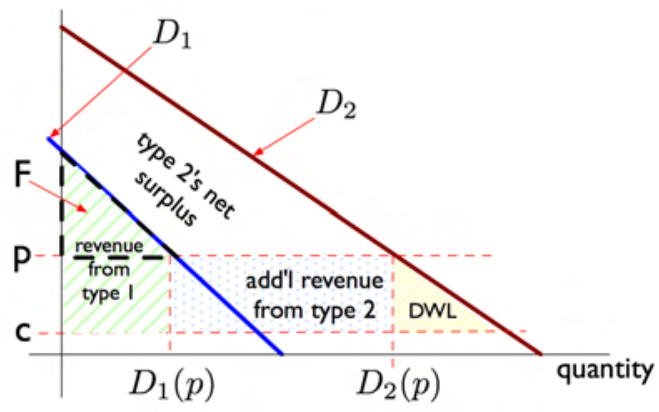

(a) Single two-part tariff.

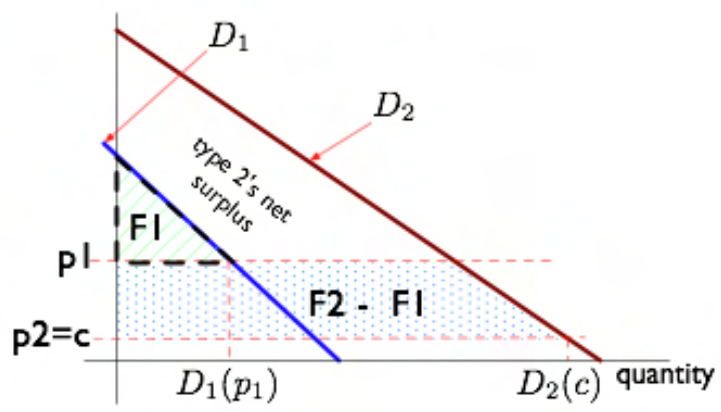

(b) Menu of two two-part tariffs.

Figure 4: Consumer choice and consumer/firm surpluses under two-part tariffs, offered in market with two consumer segments with demand functions $D_{1}(p)$ and $D_{2}(p)$.

All offers must meet the individual-rationality constraints. To make the result non-trivial, we assume it is always optimal for the firm to meet the IR constraint for type 1 , that is, $\lambda>\hat{\lambda}$. The threshold $\hat{\lambda}$ for serving both types is obtained by balancing the revenue loss from type 2 customers with the additional revenue from type 1 . Formally, with $p_{1}^{*}$ as defined by Eq. 2,

$$
\hat{\lambda}=\operatorname{sol} .\left[\lambda\left(S_{1}\left(p_{1}^{*}\right)+\left(p_{1}^{*}-c\right) \cdot D_{1}\left(p_{1}^{*}\right)\right)=(1-\lambda)\left(S_{2}\left(p_{1}^{*}\right)-S_{1}\left(p_{1}^{*}\right)\right)\right]
$$

Lemma 1 (Optimal menu of 2-part tariffs) For $\lambda>\hat{\lambda}$, the optimal menu $\left[\left(F_{1}^{*}, p_{1}\right)^{*} ;\left(F_{2}^{*}, p_{2}^{*}\right)\right]$ is

$$
\begin{aligned}
p_{1}^{*} & =\text { sol. }\left[\frac{(1-\lambda)}{\lambda}=\frac{-(p-c) D_{1}^{\prime}(p)}{\left(D_{2}(p)-D_{1}(p)\right)}\right] \\
F_{1}^{*} & =\int_{p_{1}^{*}}^{\bar{p}_{1}} D_{1}(\tau) d \tau \\
p_{2}^{*} & =c \\
F_{2}^{*} & =F_{1}^{*}+\int_{c}^{p_{1}^{*}} D_{2}(\tau) d \tau
\end{aligned}
$$

The menu of two-part tariffs implements price discrimination by charging type 2 customers a higher fixed fee $F_{2}$. It increases profits by capturing a greater amount of tradeable surplus, accomplished by giving type 2 customers a low usage fee $p_{2}=c$, and capturing part of this surplus through $F_{2}$. Assumption 1 guarantees uniqueness of the $p_{1}^{*}$ solution in Eq. 4, though in general this non-linear equation may be 
hard to solve. If $D$ is linear, a closed-form value for $p_{1}^{*}$ is easy to derive.

$$
\text { If } D_{i}(p)=\frac{1}{b_{i}}\left(a_{i}-p\right) \text {, then } p_{1}^{*}=\frac{(1-\lambda)\left(a_{2} b_{1}-a_{1} b_{2}\right)}{\lambda b_{2}+(1-\lambda)\left(b_{1}-b_{2}\right)}
$$

where $a_{2}>a_{1}$ and $b_{2}<b_{1}$. The linear demand case makes evident the following property that also holds under the general demand function $D(p)$.

Corollary $1 p_{1}^{*}$ decreases as $\lambda$ increases, and $p_{1}^{*} \rightarrow c$ as $\lambda \rightarrow 1$.

The intuition behind this result is straightforward. Under the optimal 2PT menu, type 1 customers are assigned an inefficient consumption level $\left(D_{1}\left(p_{1}^{*}\right)<e_{1}\right)$, while type 2 consumers enjoy the ideal consumption. But the firm must give each type 2 customer an "information rent" (equal to the surplus she would have obtained by pretending to be type 1 ), while there is a deadweight loss for each type 1 customer. Designing the optimal 2PT menu essentially involves a tradeoff between the deadweight loss and information rent. As $\lambda$ increases, this deadweight loss becomes increasingly important to capture, which is accomplished by lowering $p_{1}^{*}$ (thereby increasing consumption, and transfering the usage-based revenue to the fixed fee).

For the general case, the firm's optimal profit, total quantity consumed, total surplus from trade, consumer surplus, and deadweight loss under the 2PT menu are stated below.

$$
\begin{aligned}
\pi_{2}^{*} & =\lambda\left(S_{1}\left(p_{1}^{*}\right)+\left(p_{1}^{*}-c\right) D_{1}\left(p_{1}^{*}\right)\right)+(1-\lambda)\left(S_{2}(c)-\left(S_{2}\left(p_{1}^{*}\right)-S_{1}\left(p_{1}^{*}\right)\right)\right) \\
T Q_{2} & =\lambda D_{1}\left(p_{1}^{*}\right)+(1-\lambda) D_{2}(c) \\
T S_{2} & =\lambda\left(S_{1}\left(p_{1}^{*}\right)+\left(p_{1}^{*}-c\right) D_{1}\left(p_{1}^{*}\right)\right)+(1-\lambda)\left(S_{2}(c)\right) \\
C S_{2} & =(1-\lambda)\left(S_{2}\left(p_{1}^{*}\right)-S_{1}\left(p_{1}^{*}\right)\right) \\
D W L_{2} & =\lambda \int_{c}^{p_{1}^{*}} D_{1}(\tau) d \tau
\end{aligned}
$$

\subsection{Single three-part tariff beats menu of two-part tariffs}

Lemma 1 describes the optimal 2PT menu offered to a market with two customer segments. Given the optimization procedure described in the previous section, this optimal menu can be characterized by $p_{1}^{*}$, 


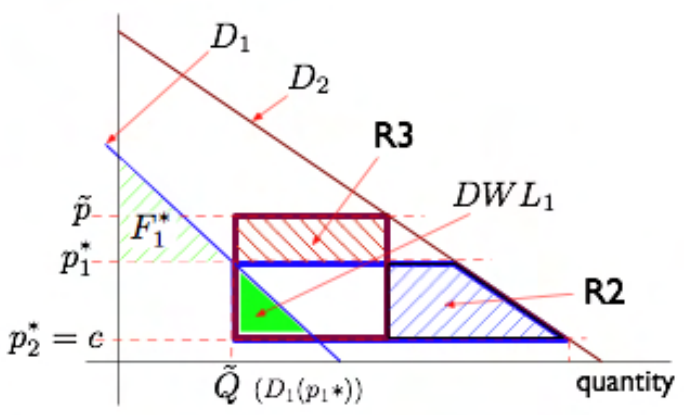

(a) Relatively low $\lambda$.

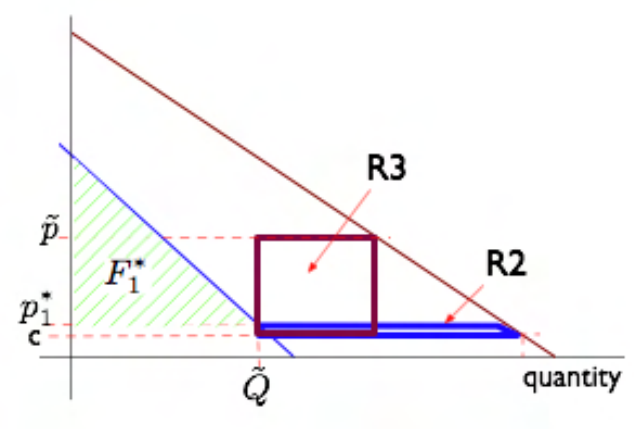

(b) High $\lambda$.

Figure 5: Relative profit from type 2 customers for the optimal 2PT menu and the construced single 3PT.

the unit fee targeted to type 1 customers. We will employ this optimal 2PT design in constructing an associated (suboptimal) single, more profitable, 3PT $(\tilde{F}, \tilde{Q}, \tilde{p})$ (with fixed fee $\tilde{F}$, an allowance $\tilde{Q}$, and unit fee $\tilde{p}$ for above-allowance consumption). To make the problem non-trivial, $\lambda$ is large enough that both types are served under either tariff mechanism.

First, we ensure that the 3PT fetches exactly the same profit $(\tilde{F})$ from type 1 customers (as well, the same level of consumption and deadweight loss) as the optimal 2PT menu. Under the latter, each type 1 customer has consumption $D_{1}\left(p_{1}^{*}\right)$, yielding a profit contribution equal to the sum of the fixed fee $F_{1}^{*}$ and the margin $\left(p_{1}^{*}-c\right) D_{1}\left(p_{1}^{*}\right)$ from usage-based fees. So, for the single three-part tariff, we club both revenue sources into the fixed fee $\tilde{F}$ and set the "free allowance" $\tilde{Q}$ to be exactly the type 1 customer's consumption under the $2 \mathrm{PT}$ menu. Hence, we get $\tilde{Q}=D_{1}\left(p_{1}^{*}\right)$, and $\tilde{F}=\left(F_{1}^{*}+\left(p_{1}^{*}-c\right) \tilde{Q}\right)+c \tilde{Q}$. Please see Figure 5(a) for a graphical illustration of the derivation. Finally, set the unit fee $\tilde{p}$ in the 3PT to some amount that induces type 2 customers to exceed the allowance level $\tilde{Q}$. This usage-based revenue from type 2 customers is modeled via the truncated demand function $\tilde{D}_{2}(p ; Q)=\left(D_{2}(p)-Q\right)$. The optimal price $\tilde{p}$ corresponding to the allowance $\tilde{Q}$ is

$$
\tilde{p}(\tilde{Q})=\arg \max _{p}(p-c)\left(D_{2}(p)-\tilde{Q}\right)
$$

The result that the single $3 \mathrm{PT}$ can be more profitable than the optimal $2 \mathrm{PT}$ menu abides the following logic (please see the Appendix for a formal proof), comparing the two profits $\tilde{\pi}_{3}$ and $\pi_{2}^{*}$. By construction, the $2 \mathrm{PT}$ menu and the 3PT extract identical profit $(\tilde{F})$ from type 1 customers, but differ 
in the additional profit from type 2 customers. With the 2PT menu, type 2's profit contribution exceeds type 1 's by the region $R_{2}$ depicted in Figure 5(a). For the 3PT, the excess profit extraction from type 2 customers is the region $R_{3}$. Thus, $\left(\tilde{\pi}_{3}>\pi_{2}^{*}\right)=\left(R_{3}-R_{2}\right)$. Recall that as $\lambda$ increases (i.e., the distribution is more left-skewed), the optimal 2PT menu sets a lower $p_{1}^{*}$ (Corollary 1 ). In the limit, as $\lambda$ approaches $1, p_{1}^{*}$ approaches $c$ (see Figure 5(b)). But this makes type 2 customers less willing to pay the higher fixed fee $F_{2}$, making it harder for the 2PT menu to effectively discriminate. Hence increase in $\lambda$ reduces $R_{2}$, the additional profit extracted from type 2 . The single 3PT, on the other hand, maintains the ability to capture additional surplus of the higher-type customers, as shown in Figure 5(b). Thus, there must exist some intermediate value $\bar{\lambda}$ such that $R_{3}>R_{2}$ for all $\lambda>\bar{\lambda}$. This condition can alternately be stated as $\exists \alpha$ such that $\frac{\lambda}{1-\lambda}>\alpha$.

Theorem 1 Under Assumption 1, a single 3PT beats the optimal 2PT menu for all $\lambda$ such that $\frac{\lambda}{1-\lambda}>\alpha$. A (non-optimal) 3PT with this property is

$$
\left(\tilde{F}=F_{1}^{*}+\left(p_{1}^{*}-c\right) \tilde{Q}, \quad \tilde{Q}=D_{1}\left(p_{1}^{*}\right), \quad \tilde{p}=\arg \max _{p}(p-c)\left(D_{2}(p)-\tilde{Q}\right)\right)
$$

where $p_{1}^{*}$ describes the optimal 2PT menu.

This analysis illuminates how the distribution of types (specifically, the left-skewness) impacts the relative superiority of $2 \mathrm{PT}$ menu vs single $3 \mathrm{PT}$. Recall, from $\S 3.1$, that the $2 \mathrm{PT}$ menu assigns belowefficient consumption to type 1 customers, causing a deadweight loss $D W L_{2}$ which is linear in $\lambda$ (see Eq. 12). For smaller $\lambda, D W L_{2}$ is small relative to the information rent needed to induce type 2 customers to pay the higher fixed fee. However, as $\lambda$ increases, $D W L_{2}$ becomes more important to capture. In fact, both the 2PT menu and 3PT capture more of $D W L_{2}$ through an increase in fixed fee ( $\tilde{F}$ for $3 \mathrm{PT})$. However, for the $2 \mathrm{PT}$ menu, the reduction in $p_{1}$ negatively impacts surplus extraction from type 2 customers, whereas the single 3PT does not face this harsh trade-off because the instrument (unit fee $\tilde{p}$ ) that targets the excess surplus of type 2 customers is not constrained by the type 1 customers' participation constraint.

Since the suboptimal 3PT defined above beats the optimal 2PT menu, so will the optimal 3PT (specified in the following section). Theorem 1 confirms the intuition provided in the various examples 
(§2) that a lean, single, three-part tariff outperforms the optimal 2PT menu when the distribution of consumer types is sufficiently skewed towards the left. While this result is robust to the nature of demand function, it is useful to consider specific demand functions in order to see that the $\alpha$ threshold is reasonable and feasible in practice.

Corollary 2 Let $D_{i}(p)=\left(a_{i}-p\right)$, with $c=0$. Then the single 3PT has higher profit than the optimal 2PT menu for all $\alpha>\frac{\sqrt{5}}{1-\sqrt{5}} \approx \frac{0.7}{0.3}=2.3$.

The condition for superiority of the single 3PT is simply that there are at least 2.3 times as many type 1 customers as type 2 customers. This is reasonable because in many markets, the number of the low-type customers (or "light" users) is far larger than the number of high-type customers (or "heavy" users). The Corollary applies to the case of parallel linear demands $\left(b_{1}=b_{2}\right)$, and it is easily seen that the condition would be further relaxed under the more reasonable property $b_{2}<b_{1}$.

\subsection{Optimal Single Three-Part Tariff}

Theorem 1 proved that a "suboptimal" single three-part tariff can outperform the optimal 2PT menu when the distribution of types is sufficiently left-skewed. Now consider the design of the optimal single 3PT $(F, Q, p)$. In general, the optimal tariff may either serve both types or just the high type. However, we compute the 3PT tariff assuming both types are served: sometimes this will underestimate the optimal profit, but the difference will not be material to our results. This is because when it is optimal to serve the high type only, the single three-part tariff and single two-part tariff both generate identical profit, while the 2PT menu does better (if $\lambda>\hat{\lambda}$ ).

The firm's revenue from a customer who consumes $q$ units under this tariff is $T(q)=F+p(q-Q)^{+}$. A type $i$ customer's optimal consumption under this tariff is

$$
\left\{\begin{array}{cll}
Q & \text { if } \quad D_{i}(p) \leq Q \quad\left(\equiv v_{i}(Q) \leq p\right) \\
D_{i}(p) & \text { if } \quad D_{i}(p)>Q
\end{array}\right.
$$

First, we establish that we only need search for tariffs under which a type 1 customer uses exactly the allowance level $Q$ (in other words, $Q \geq D_{1}(p)$ ). 


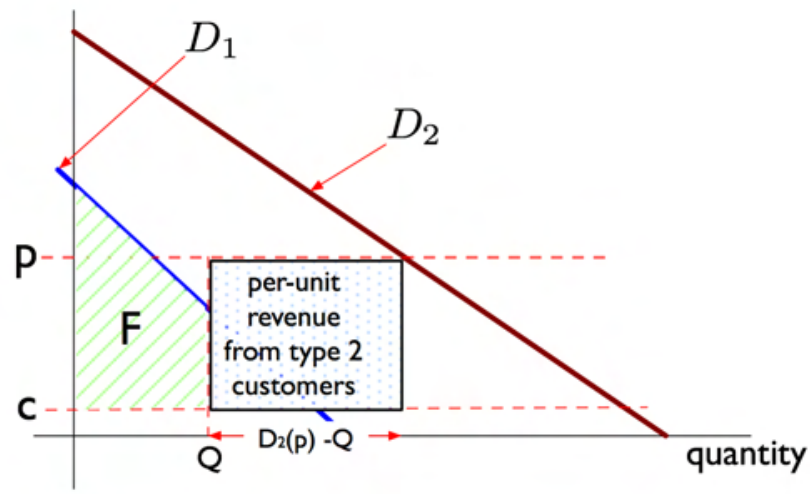

Figure 6: Revenue and consumer choice under three-part tariff. Type 1 buyers consume $Q$ units while type 2 buyers use an additional $D_{2}(p)-Q$ units.

Lemma 2 The optimal profit over the space of $3 P T$ solutions $\left(F, Q, p: v_{1}(Q) \leq p\right)$ is as good as the overall optimal profit over all possible 3PT solutions.

With this result, it follows that given $p$ and $Q$, the firm should set $F$ to extract the entire remaining surplus of a type 1 customer,

$$
F^{*}(Q)=\int_{0}^{Q} v_{1}(q) d q
$$

earning a total profit

$$
\pi(Q, p)=F^{*}(Q, p)+(1-\lambda)(p-c)\left(D_{2}(p)-Q\right) .
$$

Moreover, corresponding to the allowance $Q$ the only additional revenue for the firm (besides the fixed fee) is revenue from type 2 consumers. This revenue may be modeled via the truncated demand function $\tilde{D}_{2}(p ; Q)=\left(D_{2}(p)-Q\right)$. Figure 6 depicts the firm's revenue and consumers' consumption choice. The optimal price $p^{*}(Q)$ corresponding to an allowance $Q$ is

$$
p^{*}(Q)=\arg \max _{p}(p-c)\left(D_{2}(p)-Q\right)
$$

This problem has the usual optimal solution given by "market power equals inverse elasticity of demand" (for demand function $\tilde{D}_{2}(p ; Q)$ ). Substituting this into the firm's profit function, and writing $D_{2}^{\prime}(p ; Q)$ for the partial derivative with respect to $p$, we get 
Lemma 3 (Optimal three-part tariff) The optimal three-part tariff $\left(F^{*}, Q^{*}, p^{*}\right)$ is obtained by optimizing the single-variable objective function $\pi(Q)$, with

$$
\begin{aligned}
Q^{*} & =\arg \max _{Q} \pi(Q)=\arg \max _{Q}\left(F^{*}(Q)-c Q+(1-\lambda)\left(p^{*}(Q)-c\right)\left(D_{2}\left(p^{*}(Q) ; Q\right)-Q\right)\right) \\
p^{*}(Q) & =\text { sol. }\left[\frac{p-c}{p}=-\frac{D_{2}(p ; Q)-Q}{D_{2}^{\prime}(p ; Q)}\right] \\
F^{*}(Q) & =\int_{0}^{Q} v_{1}(q) d q
\end{aligned}
$$

Again, uniqueness of the optimal solution is guaranteed under Assumption 1. The firm's optimal profit, total quantity consumed, and total surplus from trade under the single 3PT are:

$$
\begin{aligned}
\pi_{3}^{*} & =\int_{0}^{Q^{*}}\left(v_{1}(q)-c\right) d q+(1-\lambda)\left(p^{*}-c\right)\left(D_{2}\left(p^{*}(Q) ; Q\right)-Q^{*}\right) \\
T Q_{3} & =Q^{*}+(1-\lambda)\left(D_{2}\left(p^{*}(Q) ; Q\right)-Q^{*}\right) \\
T S_{3} & =\lambda\left(\int_{0}^{Q^{*}}\left(v_{1}(q)-c\right) d q\right)+(1-\lambda)\left(\int_{0}^{D_{2}\left(p^{*}(Q) ; Q\right)}\left(v_{1}(q)-c\right) d q\right)
\end{aligned}
$$

\subsection{Welfare Implications}

The relationship between the choice of tariff and consumer/producer surplus can be fairly complex as illustrated earlier in Example 1. Intuitively, the tariff structure that causes higher consumption should, under zero marginal cost, also generate higher total surplus. But the example stands this intuition on its head. For the given demand parameters (and with $\lambda=0.8$ ), the three-part tariff leads to higher total consumption (103.27 units vs. 101.77 for the 2PT menu), but it reduces consumer surplus (2422 vs. 2972$)$ as well as total social welfare ( 8400 vs. 8501$)$; the loss to consumers exceeds the gain to the firm. On the other hand, the 3PT generates higher consumption and higher social welfare (as well as higher profit) when $\lambda=0.7$. And for other parameter values, the single 3PT increases producer profits, consumer surplus, and total surplus.

To further illustrate this point, we restrict our model to a specific demand structure $D_{i}(p)=\left(a_{i}-b p\right)$. With this setting we can easily compute the optimal prices, profit and social surplus, leading to the following theorem. 
Theorem 2 There is an interval $\left(\lambda_{1}, \lambda_{2}\right)$ such that $\forall \lambda \in\left(\lambda_{1}, \lambda_{2}\right)$ a three part tariff is more profitable and generates more total surplus than that a menu of two two-part tariffs.

While the complete proof is in the Appendix, we note that the ordering of the two tariff structures on total consumption depends only on $b$ (specifically, $T Q_{3 P T}-T Q_{2 P T}$ if and only if $b<1$ ) while the ordering on total surplus depends on $\lambda$ (specifically, $T S_{3 P T}>T S_{2 P T}$ if and only if $\lambda<\sqrt{\frac{1}{2}}$ ). This means that compared with an optimal menu of two $2 P T$, a single $3 P T$ may change total consumption and total surplus either in the same or opposite direction.

The relationship between total consumption and social welfare can be further complicated in presence of regulatory preference over the total amount of consumption. There are many economic situations where the social welfare is a function of the total consumption as well as the total surplus (the sum of consumer surplus plus profits). In other words, if we let $S W=f(T S, T Q)$, then $\frac{\partial f}{\partial T S}$ is always positive (this represents the common view of social welfare). However, $\frac{\partial f}{\partial T Q}$, can be either positive or negative. We now provide examples for such situations.

First consider the case of a public utility selling water (or, for that matter, wireless spectrum). Since water is an exhaustible resource with an uncertain rate of renewal, it is reasonable to assume that for the same level of profits the public utility prefers to sell less water than more water (this is actually observed in real life with public utilities encouraging people to conserve water). It is also reasonable to assume that the social planner would prefer a pricing mechanism that encourages conservation (see Krause et al. (2002) for a discussion of the role of consumer heterogeneity and non-linear pricing in water conservation). This is an example for the case $\frac{\partial f}{\partial T Q}<0$. From Theorem 2 (please see Eq. 21 in the proof), we can easily see that when $b>1$, a $3 \mathrm{PT}$ tariff is more consistent with conservation efforts than a $2 \mathrm{PT}$

On the other hand, sometimes $\frac{\partial f}{\partial T Q}>0$. For example, there are several studies on the effect of different nonlinear pricing mechanisms of drugs on the level of prescription compliance in heterogenous population of patients (Goldman et al., 2006). In these studies, the population of patients is divided into finite number of types with different demand functions. Normally, these types corresponds to different risk factors, for example low and high risk types. Increasing the total compliance level of the patients (total consumption of the drug) has social benefits that cannot be captured directly by the total surplus 
(i.e. $\frac{\partial f}{\partial T Q}>0$ ). The pharmaceutical companies can charge a certain price per refill (linear pricing), a fixed fee plus a price per refill (two-part tariff), or a fixed fee that covers a certain number of refills with additional charge per refill for refills in excess of this number (i.e, a three-part tariff). Based on our earlier analysis, if $b<1$ and $\lambda$ is in a certain range, then 3PT can yield more profits and induce higher level of compliance, than linear and two-part tariffs. In fact in this case, the 3PT can be more socially desirable the 2PT even if it yields lower total surplus than 2PT.

The two above examples and Eq. 20- 21 suggest that when $b<1$ and $\frac{\partial f}{\partial T Q}>0$, it is possible that the $3 \mathrm{PT}$ is more socially desirable even if $\lambda$ is such that $T S_{3 P T}<T S_{2 P T}$. More generally, $3 P T$ can be more desirable than $2 P T$, even if $\lambda \notin\left(\lambda_{1}, \lambda_{2}\right)$. Similarly, when $b>1$ and $\frac{\partial f}{\partial T Q}<0$, a $2 \mathrm{PT}$ can be socially more desirable even if $\lambda \in\left(\lambda_{1}, \lambda_{2}\right)$.

\section{Extension to Multiple Consumer Types}

In $\S 3$ we showed that the single 3PT dominates the 2PT menu (Theorem 1 ). The result was derived with a very general demand function, hence is robust to the nature of individual demand preferences. However our analysis was restricted to $I=2$ customer types, and it might be argued that small $I$ handicaps the 2PT menu by limiting possibilities for pooling ex ante types into ex post segments. So, a natural question is: does the result hold for large $I$ ? When $I$ is large, the firm has greater flexibility in designing the $2 \mathrm{PT}$ menu: there can be upto $I$ items in the menu, hence $2^{I-1}$ ways to partition customers into pools (note: type $i-1$ must pick either the same item as type $i$ or the one immediately lower). In this section we show that a single three-part tariff produces higher profit even when $I$ is large, so long as the distribution of consumer types is sufficiently left-skewed. And when a single 3PT cannot succeed, usually a very short menu of 3PT can beat even a complete-sorting 2PT menu with several items. We also elaborate on the intuition provided in $\S 2$ that this superior 3PT can be designed with relatively minimal information about demand preferences.

\subsection{Analysis}

Suppose there are $I$ agents with marginal valuations $v_{i}(q)$ and demand where $v_{i}(q)<v_{i+1}(q)$ for all $i, q$. Let $D_{i}(p)$ represent type $i$ 's demand at unit price $p$, and $\lambda$ the weight of agent $i$. For sake of 
exposition, let $c=0$. Since the profit performance of a 2PT menu (relative to that of 3PTs) is best when the $2 \mathrm{PT}$ menu implements complete sorting, we shall restrict attention to this case.

Lemma 4 The optimal, fully separating, menu of two part tariffs is given by pairs $\left[\left(F_{i}^{*}, p_{i}^{*}\right)\right]$ where

$$
\begin{aligned}
p_{i}^{*} & =\text { Sol. }\left[p=\left(\frac{\sum_{j=i+1}^{I} \lambda_{j}}{\lambda_{i}}\right) \frac{1}{-D_{i}^{\prime}(p)}\left(D_{i+1}(p)-D_{i}(p)\right)\right] \\
F_{i}^{*} & =p_{i}^{*} v_{i}\left(D_{i}\left(p_{i}^{*}\right)\right)+\int_{p_{1}}^{\bar{p}_{1}} D_{1}(\tau) d \tau+\int_{p_{2}}^{p_{1}} D_{2}(\tau) d \tau+\cdots+\int_{p_{i}}^{p_{i-1}} D_{i-1}(\tau) d \tau
\end{aligned}
$$

with $p_{1}>p_{2}>\cdots>p_{I}=0$.

For the case of $I=2$ discussed in $\S 3$, the optimal separating $2 \mathrm{PT}$ is $\left[\left(F_{1}^{*}, p_{1}\right)^{*} ;\left(F_{2}^{*}, p_{2}^{*}\right)\right]$ where

$$
p_{1}^{*}=\frac{1-\lambda}{\lambda} \frac{D_{2}\left(p_{1}^{*}\right)-D_{1}\left(p_{1}^{*}\right)}{-D_{1}^{\prime}\left(p_{1}^{*}\right)}
$$

Recall, for the $I=2$ case, the essence of our result (Theorem 3): when $\lambda$ is large, the 2PT menu optimization sets $p_{1}^{*}$ low, and this permits the design of a single 3PT which extracts more additional surplus from type 2 customers (and identical profit from type 1 customers). Now the statement " $p_{1}^{*}$ is low enough" can be translated, via Eq. 18, into a condition on $\frac{\lambda}{1-\lambda}$ being large enough, which we interpreted as the overall distribution being sufficiently left-skewed.

For the general case of $I>2$ the essential logic is similar. If, for some $i=k<I, p_{k}^{*}$ is small enough, then we can repeat and generalize the logic of the $I=2$ case as follows. For customer types $k+1$ to $I$, we find a single $3 \mathrm{PT}\left(\tilde{F}_{k}, \tilde{Q}_{k}, \tilde{p}\right)$ which generates more profit from these consumers than the entire old submenu of $2 \mathrm{PT}$ s consisting of $\left[\left(F_{k+1}^{*}, p_{k+1}^{*}\right) \ldots\left(F_{I}^{*}, p_{I}^{*}\right)\right]$. For customer types $1 \ldots k$, we generate 3PT items from the corresponding $2 \mathrm{PT}$ items as in $\S 3.2$ : for each $2 \mathrm{PT}\left(F_{i}^{*}, p_{i}^{*}\right)$ we create a 3PT $\left(\tilde{F}_{i}, \tilde{Q}_{i}, \tilde{p}\right)$ with $\tilde{Q}_{i}=D_{i}\left(p_{i}^{*}\right)$ and $\tilde{F}_{i}=F_{i}^{*}+p_{i}^{*} \tilde{Q}_{i}$. Notice that the unit fee $\tilde{p}$ is the same in all the 3PT menu items. Thus the overall 3PT menu is shorter and less ornate (fewer instruments) than the optimal 2PT menu, and it fetches higher profit.

Now, note that requiring $p_{k}^{*}$ to be small enough is the same as requiring

$$
H(k)=\frac{\lambda_{k}}{\sum_{j=k+1}^{I} \lambda_{k}}
$$


to be large. The term $H(k)$ can be interpreted as a discretized hazard rate for the distribution of types. Furthermore, "large enough" is relative to a threshold $\alpha_{k}$. If we were to design the true optimal 3PT, this $\alpha_{k}$ will require knowledge of all $a_{i}$ 's for $i=k \ldots I$. But to design only this "superior but suboptimal" 3PT, the $\alpha_{j}$ threshold is independent of the $a_{i}{ }^{\prime}$ s for $i=k+2 \ldots I$. If this condition holds at $k=1$, then this is where we "shorten" the menu. If the lowest $k$ where this condition holds is $k=5$, then again, there would be 5 items in the 3PT menu (relative to at least 6 in the 2PT menu).

Theorem 3 Suppose the distribution is such that the optimal 2PT is fully separating. Then, for every $i, 1 \leq i<I, \exists \alpha_{i}\left(a_{1}, \cdots, a_{I}, b\right)$ such that if $H(i)>\alpha_{i}$, then a (shorter) menu of three-part tariff $\left(\hat{F}_{j}, \hat{Q}_{j}, \hat{p}\right)$, for $j=1, \cdots i$, yields higher profit than the optimal two-part tariff menu consisting of $I$ items. In particular, if $H(1)>\alpha_{1}$, then a single $3 P T,\left(\tilde{F}_{1}, \tilde{Q}_{1}, \tilde{p}\right)$, will beat the entire menu of $I$ two-part tariffs.

The result as stated above is framed with problem parameters such that the optimal $2 \mathrm{PT}$ menu is fully separating. However, as illustrated earlier in Example 3, the result is even stronger if we consider that the optimal 2PT menu may implement partial pooling. In this case redefine the set $I$ to be the set of types that are strictly targeted with each menu item, and modify the distribution of types to take pooling into account. Then, the result holds so long as the left-skewness property $\left(H(i)>\alpha_{i}\right.$ for some i) holds with respect to the post-pooling distribution.

The significance of Theorem 3 is three-fold. First, it generalizes to $I>2$ types the notion of "sufficiently left-skewed distribution" and provides a quantitative measure of it in terms of the discretized hazard rate $H(i)$. Intuitively, the goal is to pick the smallest $j$ that satisfies this property. When $k=1$, then a single 3PT beats the optimal 2PT menu. Moreover, when $k \geq 2$, the result specifies the number of items in a 3PT menu that is sufficient to beat the optimal 2PT menu.

Second, note that for $i<k$, the seller uses $\tilde{p}_{k}$, the over-allowance unit price, simply as a tool to stop higher types from pretending to be lower types. In particular, the seller can choose a single $\tilde{p}$ as long as it is high enough to ensure proper self selection. This is consistent with the observation that actual menus of three-part tariffs use an single $\tilde{p}$. It is also consistent with the empirical finding in Lambrecht and Skiera (2006) that consumer's choice of items on a three-part menu is not sensitive to $\tilde{p}$. 
The third significant aspect of the more austere 3PT menu (relative to the 2PT menu) is the impact of "hidden costs" of price discrimination that are otherwise not factored into the tariff optimization. Specifically, discriminating between customers by inducing self-selection out of a multi-item menu (as in the 2PT menu) imposes two kinds of costs on the firm. First, for setting the optimal menu the firm must collect extremely fine-grained data about customer distribution and preferences. Second, once customers have been pooled into segments, the firm incurs segment developments costs because it must advertise and target each segment. These costs can be substantial, as illustrated in Dhebar (1993). Therefore, not only is the leaner 3PT menu more profitable (in terms of variable revenues and costs), it has also subsantially lower administrative costs.

\subsection{Discussion of Assumptions}

All our results are derived under a very general demand function representing (for each consumer type) consumer valuations for multiple units of the product. Specifically, we require only a downward sloping demand function with positive elasticity of demand. The novel aspect of our analysis is in how we model the density of consumer types. Prior models of nonlinear pricing assume either a continuous distribution - an abstraction that enables the use of calculus, but requires a "smoothness" constraint such as non-decreasing hazard rate (Mussa and Rosen, 1978), increasing generalized failure rate (Lariviere, 2006), or non-decreasing demand elasticity (Maskin and Riley, 1984)—or a discrete set with just two consumer types (simplifies computation but limits applicability and generality of results). In reality, however, many applications fit neither extreme: there is a large number of discrete consumer segments, and the density function has multiple sharp peaks and valleys that violate the smoothness constraint (see Figure 2(b), Example 3). Often such data cannot reasonably be fitted by a single distribution, so more recently researchers and practitioners have been applying mixture distributions, fitting the data with a convex combination of two or more distributions (Wendel and Kamakura, 2000, Ch. 6).

Our results are based on a formulation which allows for an arbitrarily large number of discrete consumer segments. The density of types need not be monotonic, continuous or with an increasing hazard rate. The only condition for our results is that the distribution is left-skewed which means that the hazard rate $H(k)$ is high enough for some $k$. This requirement is generally satisfied, even more so 
because we have a fairly loose definition of left-skewness (i.e., flexibility in choosing the left and right

partitions). This follows the general intuition that in most markets there are more consumers at the "low end" than at the "high end" - our specific assumptions in this case are confirmed using real-world data from one of the largest telecommunications operators. Moreover the skewness need not even be present in the exogneous distribution; it should be present, as is generally to be expected, in the endogenous segments created when the 2PT menu does not implement complete sorting (recall Example 3, and the discussion following Theorem 3).

\section{Conclusion}

We have compared the performance of 3PT menus with 2PT menus in terms of profits and social welfare. We find, surprisingly, that a less ornate tariff-a leaner, simpler menu of 3PT (and quite often, just a single three-part tariff)—can be more profitable than more ornate 2PT menus. Our analysis reveals a novel insight about the relationship between a tariff structure's degree or ornateness and ability to improve profit through price discrimination: the source of ornateness matters. To elaborate on this point, compare, first, a single two-part tariff with a single three-part tariff. The 3PT is more ornate, and its ornateness derives from one additional dimension for price discrimination $(Q)$. Now compare a four-item 2PT menu with a two-item 3PT menu. The 2PT menu is more ornate, but because it places more items on the menu. However, its ability to price discriminate is limited by consumers' self-selection constraints. These constraints make the more ornate 2PT menu more complex to design (the firm needs to optimize more decision variables) and leave the firm with fewer degrees of freedom. From a managerial perspective, what is significant here is that the 3PT structure not only produces higher profit, but it requires less information and has lower managerial and decision complexity.

We note that the profit advantage of 3PT over 2PT can be amplified under any of the following additional characteristics. First, the presence of segment development and data collection costs that increase with the length of the menu. Second, when regulatory preference favors conservation (as discussed in $\S 3.4$ ), or when there are negative externalities due to congestion effects (Masuda and Whang, 2006). Third, when there are some time-inconsistent (naïve) consumers with uncertain demand who are unaware of the fact that they are time inconsistent (Malmendier and Della Vigna, 2005). 
Fourth, when consumers exhibit a bias toward tariffs with high allowance, preferring tariffs with high $\hat{Q}$ independent of their consumption patterns (Lambrecht and Skiera, 2006).

Our analysis of MPTs is a stepping stone to a study of broader issues related to the use of these tariff structures. A common characteristic of IT and telecommunications products that employ MPTs is that they require a customer to sign a long-term contract covering a period for which customers are uncertain about their demand pattern. Consequently, customers must make a purchase decision while they are uncertain about their valuation for the service (but they choose consumption quantity, within their chosen tariff, after resolving uncertainty). This phenomenon also permeates many other businesses where modern information technologies such as e-ticketing enable firms to sell products (such as concert seats, airline travel, hotel rooms etc.) far in advance of consumption, leaving customers uncertain about product valuation at the time of purchase (Shugan and Xie, 2000; Xie and Shugan, 2001). Selling late, on the other hand, allows customers to (privately) get more information about their future valuations, increasing the information asymmetry and leaving the seller with an information disadvantage. In both classes of examples, the firm's choice of when to sell affects the extent of information asymmetry in the pricing problem. Lambrecht and Skiera (2006) noted that when the demand of the individual consumer is uncertain, then any separation between decision making (what tariff to sign for) and consumption will have different implications under 2PT and 3PT structures. More specifically, if the uncertainty is due to a demand shock that is symmetric (the demand curve of the consumer moves to the right and to the left of some average value), then under 2PT the consumer will incur the same charges under certain and uncertain demand. Under 3PT however, the charges under certain and uncertain demand will be different since going below $\tilde{Q}$ has different cost than going above $\tilde{Q}$. In a companion paper to this one, we explore the relationship between the tariff structure used for price discrimination, the degree of information asymmetry, and the timeline of a contract (i.e., "who knows what, and when they know it"). The results derived in this paper are directly applicable to this broader problem because the optimization problem of MPT design under demand uncertainty can be reduced to price optimization under deterministic demand.

Acknowledgements We thank our colleagues Rachel Chen and Vidyanand Choudhary for helpful comments on an earlier draft. 


\section{References}

Armstrong, M. (2006): "Recent Developments in the Economics of Price Discrimination," Advances in Economics and Econometrics:Theory and Applications; Forthcoming, Ninth World Congress, Cambridge Univeristy Press.

Clay, K. B., D. S. Sibley, and P. Srinagesh (1992): "Ex Post vs. Ex Ante Pricing: Optional Calling Plans and Tapered Tariffs," Journal of Regulatory Economics, 4, 115-38, available at http://ideas.repec.org/a/kap/regeco/v4y1992i2p115-38.html.

Dhebar, A. (1993): “Cambridge Software Corp." Harvard Business School.

Faulhaber, G. R. And J. C. Panzar (1977): "Optimal Two-Part Tariffs with Self-Selection," Tech. rep., Bell Laboratories Economic Discussion Paper No. 74.

Goldman, D., G. Joyce, And P. Mandic (2006): "Varying Pharmacy Benefits with Clinical Status: The Case of Cholesterol-Lowering Therapy," The American Journal of Managed Care, 12, 21-28.

Kolay, S. And G. Shaffer (2003): "Bundling and Menus of Two-Part Tariffs," Journal of Industrial Economics, 51, 383-403.

Krause, K., J. Chermak, And D. Brookshire (2002): "The Demand for Water: Consumer Response to Scarcity," The Journal of Regulatory Economics, 23, 167-191.

Lambrecht, A. And B. Skiera (2006): "Paying Too Much and Being Happy About It: Existence, Causes, and Consequences of Tariff-Choice Biases," Journal of Marketing Research, forthcoming.

Lariviere, M. (2006): "A Note on Probability Distributions with Increasing Generalized Failure Rates," Operations Research, 54, 602-604.

Leland, H. E. AND R. A. Meyer (1976): “Monopoly Pricing Structures with Imperfect Discrimination," The Bell Journal of Economics, 7, 449-462. 
Malmendier, U. M. and S. Della Vigna (2005): "Overestimating Self-Control: Evidence from the Health Club Industry," Tech. rep., Stanford Research Paper No. 1880.

Maskin, E. AND J. Riley (1984): "Monopoly with Incomplete Information," Rand Journal of Economics, 15, 171-194.

Masuda, Y. And S. Whang (2006): "On the Optimality of Fixed-up-to Tariff for Telecommunication Service," Information Systems Research, 17.

Miravete, E. J. (1996): "Screening Consumers Through Alternative Pricing Mechanisms," Journal of Regulatory Economics, 9, 111-132.

Mussa, M. And S. Rosen (1978): "Monopoly and Product Quality," Journal of Economic Theory, $18,301-317$.

Shugan, S. M. And J. XIE (2000): “Advance Pricing of Services and Other Implications of Separating Purchase and Consumption," Journal of Service Research, 2, 227-239.

Sundararajan, A. (2004): "Nonlinear Pricing of Information Goods," Management Science, 50, $1660-1673$.

Wendel, M. And W. Kamakura (2000): Market Segmentation: Conceptual and Methodological Foundations, Dordrecht, The Netherlands: Kluwer Academic Publishers.

Wilson, R. B. (1993): Nonlinear pricing, New York: Oxford University Press.

Xie, J. And S. M. Shugan (2001): “Electronic Tickets, Smart Cards, and Online Prepayments: When and How to Advance Sell," Marketing Science, 20, 219-243. 


\section{A Technical Details}

Proof of Lemma 2. Suppose that the optimal design fails the property, that is there is a tariff $(\hat{F}, \hat{Q}, \hat{p})$ which generates strictly higher profit and has $\hat{p}<v_{1}(Q)$. We show this is a contradiction. Let $\tilde{q}_{1}=v_{1}(\hat{p})$, and let $\tilde{F}_{1}=\int_{0}^{\tilde{q}_{1}} v_{1}(\tau) d \tau$. Then, the menu $\left(\tilde{F}_{1}, \tilde{q}_{1}, \hat{p}\right)$ will yield the same profits as $(\hat{F}, \hat{Q}, \hat{p})$ without violating the IR and IC constraints.

Proof of Theorem 1. With the 2PT menu, type 2's profit contribution exceeds type 1's by

$$
R_{2}=\int_{c}^{p_{1}^{*}} D_{2}(\tau) d \tau-\left(p_{1}^{*}-c\right) D_{2}(\tilde{p})
$$

while for the 3PT, the excess profit contribution is

$$
R_{3}=\max _{p}\left(p-p_{1}^{*}\right)\left(D_{2}(p)-\tilde{Q}\right)
$$

For the 2PT menu, Eq. 4 yields

$$
p_{1}^{*}=c+\frac{1-\lambda}{\lambda} \frac{D_{2}\left(p_{1}^{*}\right)-D_{1}\left(p_{1}^{*}\right)}{-D_{1}^{\prime}\left(p_{1}^{*}\right)}
$$

whence evident that $p_{1}^{*} \rightarrow c$ as $\lambda \rightarrow 1$ (note that the term $D_{1}^{\prime}\left(p_{1}^{*}\right)$ does not approach 0 in the limit, because the inverse function $v_{i}$ is differentiable). Therefore,

$$
\lim _{\lambda \rightarrow 1} R_{2} \quad \rightarrow 0
$$

because the lower and upper bounds of the integral converge. On the other hand, while $R_{3}=0$ for $\lambda=1, R_{3}$ remains positive as $\lambda$ approaches 1 . Hence $\pi_{3}^{*}>\pi_{2}^{*}$ for $\lambda>\hat{\lambda}$.

Proof of Corollary 2. With this specific demand function, we get closed-form values for the optimal 
tariffs under the two mechanisms:

\begin{tabular}{|c|c|}
\hline menu of two-part tariffs & subopt 3PT tariff \\
\hline$p_{1}^{*}=\frac{1-\lambda}{\lambda}\left(a_{2}-a_{1}\right)$ & $\tilde{p}=\frac{a_{2}-a_{1}}{2 \lambda}$ \\
\hline$D_{1}\left(p_{1}^{*}\right)=\frac{a_{1}-(1-\lambda) a_{2}}{\lambda}$ & $\tilde{Q}=D_{1}\left(p_{1}^{*}\right)$ \\
\hline & $D_{2}(\tilde{p})=\frac{a_{2}-a_{1}}{2 \lambda}$ \\
\hline & $D_{2}\left(p_{1}^{*}\right)=a_{2}-\frac{1-\lambda}{\lambda}\left(a_{2}-a_{1}\right)$ \\
\hline
\end{tabular}

As graphically depicted in Figure 5, the profit difference $\pi_{3}^{*}-\pi_{2}^{*}$ is the same as the difference $R_{3}-R_{2}$, so that

$$
\begin{aligned}
\left(R_{3}-R_{2}\right)>0 & \equiv[(2 \lambda-1)-2 \lambda(1-\lambda)] \tilde{p}^{2}>0 \\
& \equiv\left(2 \lambda^{2}-1\right)>0 \\
& \equiv \lambda>\sqrt{0.5}
\end{aligned}
$$

and the single three-part tariff wins when the ratio of low-end consumers is greater than $\approx 0.707$.

Proof of Theorem 2. The proof of theorem follows readily from simple calculations. Normalize the demand function by setting $a_{1}=1$ (and, since type 2 is the higher type, $a_{2}>1$ ) and $c=0$. Applying Eq. 8 and 13 to the current setting of linear parallel demand, we obtain

$$
\Pi_{3 P T}-\Pi_{2 P T}=-\frac{0.5\left(a_{2}-1\right)^{2}(\lambda-1)(\lambda-0.618)(1.618+\lambda)}{b \lambda(1+\lambda)}
$$

Similarly, applying Eq. 10 and 15, we obtain

$$
T S_{3 P T}-T S_{2 P T}=\frac{\left(a_{2}-1\right)^{2}(\lambda-1)\left(2 \lambda^{2}-1\right)}{2 b \lambda\left(1+\lambda^{2}\right)}
$$

Finally, from Eq. 9 and 14, we have,

$$
T Q_{3 P T}-T Q_{2 P T}=\frac{a_{2}(b-1)(\lambda-1)}{b}
$$


The claim of the theorem is immediate from the above two equations. Furthermore, $T Q_{3 P T}>$ $T Q_{2 P T}$ if and only if $b<1$. On the other hand, $T S_{3 P T}>T S_{2 P T}$ if and only if $\lambda<\sqrt{\frac{1}{2}}$. This means that when we switch from an optimal menu of two $2 P T$ to a single $3 P T$, the total consumption and the total surplus may move in the same direction or in opposite directions depending on the parameters of the problem.

Proof of Theorem 3. The proof is very similar to the proof in the case of two agents. Suppose, for $i=k, p_{k}^{*}$ is low enough, or alternatively suppose $\frac{\lambda_{k}}{\sum_{j=k+1}^{I} \lambda_{j}}$ is high enough. By Theorem 1 , we can set $\tilde{Q}=D_{k}\left(p_{1}^{*}\right), \tilde{F}=\int_{0}^{\tilde{Q}} v_{k}(\tau) d \tau$, and $\tilde{p}=\operatorname{argmax}(p-c)\left(D_{k+1}(p)-\tilde{Q}\right)$. Then, the net surplus the tariff $(\tilde{F}, \tilde{Q}, \tilde{p})$ extracts from consumer $k+1$ is higher than the net surplus the tariff $\left(F_{k+1}^{*}, p_{k+1}^{*}\right)$ extracts from the same consumer. The is due to the fact that

$$
R_{3}(k)=\left(\tilde{p}-p_{k}^{*}\right)\left(D_{k+1}(\tilde{p})-\tilde{Q}\right)>R_{2}(k)=\int_{c}^{p_{k}^{*}} D_{k+1}(\tau) d \tau-\left(p_{k}^{*}-c\right) D_{k+1}(\tilde{p})
$$

Figure 5(b) shows $R_{3}(1)$ and $R_{2}(1)$ in the case $I=2$. Furthermore, for any $k<j<I$, the net surplus the tariff $(\tilde{F}, \tilde{Q}, \tilde{p})$ extracts from consumer $j$ is higher than the surplus the tariff $\left(F_{j}^{*}, p_{j}^{*}\right)$ extracts from the same consumer. Again, this is due to the fact that

$$
R_{3}(j)=\left(\tilde{p}-p_{j}^{*}\right)\left(D_{j+1}(\tilde{p})-\tilde{Q}\right)>R_{2}(j)=\int_{c}^{p_{j}^{*}} D_{j+1}(\tau) d \tau-\left(p_{j}^{*}-c\right) D_{j+1}(\tilde{p})
$$

(Recall that for $j>k, P_{j}^{*}<P_{k}^{*}$ ) This implies that for consumers $k+1 \cdots n$, the tariff $(\tilde{F}, \tilde{Q}, \tilde{p})$ extracts more surplus than the entire submenu $\left(F_{k+1}^{*}, p_{k+1}^{*}\right) \cdots\left(F_{I}^{*}, p_{I}^{*}\right)$ 